\title{
Cryo-EM Workshop: Lectures on Cryo-EM Image Formation and 3-D Reconstruction
}

Wen Jiang, Ph.D.

Professor of Biological Sciences, Purdue University

Scientific Director, Purdue Cryo-EM Facility

wjiang@gmail.com

Cryo-EM is revolutionizing structural biology. It has been used to determine numerous

structures of viruses and protein complexes to 2-4 A resolutions and a few cases at sub- $2 \AA$ resolutions in recent years. The cryo-EM revolution has attracted many novice users who will need to learn both the principles and the hands-on skills of sample preparation, TEM

instruments, image processing and 3-D reconstruction, and structural analysis. In this Cryo-EM Workshop, I will present lectures on TEM optics, image formation, image processing, and 3-D reconstruction. In addition, I will also present our new virtual reality augmented hands-on training system CryoVR with the focus on sample plunge freezing. 